\title{
Kawasaki-Syndrom mit Makrophagenaktivierung bei einem sechsjährigen Mädchen
}

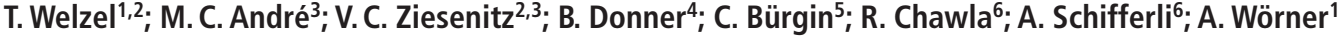

Universität Basel, Universitäts-Kinderspital beider Basel (UKBB), Basel, Schweiz; ${ }^{1}$ Pädiatrische Rheumatologie; ${ }^{2}$ Pädiatrische Pharmakologie; ${ }^{3}$ Pädiatrische Intensivstation; ${ }^{4}$ Pädiatrische Kardiologie; ${ }^{5}$ Pädiatrie; ${ }^{6}$ Pädiatrische Onkologie/Hämatologie

\section{Einleitung und Hintergrund}

Das Kawasaki-Syndrom (KS) ist eine akute febrile Vaskulitis der kleinen und mittelgroßen Arterien. Gefürchtet ist der Befall der Koronararterien (1), der lebensbedrohlich verlaufen kann. Die genaue Ätiopathologie des KS ist bis heute ungeklärt. Es scheint aber eine Immundysregulation in prädisponierten Personen ursächlich zu sein (2). Die Diagnose des KS wird anhand klinischer Kriterien gestellt (3) ( Kasten). Einen spezifischen diagnostischen Test gibt es nicht. Allerdings können bestimmte Laboruntersuchungen und die Echokardiografie bei Patienten mit Verdacht auf ein inkomplettes KS hilfreich sein (1). Typisch für die akute Phase des KS sind eine Leukozytose, eine erhöhte Blutsenkungsgeschwindigkeit (BSG), ein erhöhtes Creaktives Protein (CRP) sowie ein milder bis moderater Anstieg der Gamma-Glutamin-Transferase $(\gamma \mathrm{GT})$ und der AlaninAminotransferase (ALT) (4). Zusätzlich zeigt sich in der akuten Phase des KS häufig eine normozytäre, normochrome Anämie und in der subakuten Krankheitsphase eine Thrombozytose (4). Mittels Echokardiografie kann die Dilatation der Koronararterien beurteilt werden. Eine Erweiterung des Ramus interventrikularis anterior oder der rechten Koronararterie mit einem Z-Score $\geq 2,5$ wird dabei als Koronardilatation definiert (1).

Korrespondenzadresse

Tatjana Welzel, MD

Pediatric Rheumatology

Pediatric Pharmacology and Pharmacometrics

University Children's Hospital Basel (UKBB), University of Basel

Spitalstr. 33, CH-4056 Basel, Schweiz

E-Mail: tatjana.welzel@ukbb.ch

Tel.: +41617042252

arthritis + rheuma 2018; 38: 289-293
Mit Diagnosestellung eines KS sollte unverzüglich eine Therapie mit Immunglobulinen (IVIG) $2 \mathrm{~g} / \mathrm{kgKG} / 12$ Stunden intravenös und Acetylsalicylsäure (ASS) 80-100 mg/kgKG/24 Stunden begonnen werden $(1,5)$, um Komplikationen zu verhindern.

Das Makrophagenaktivierungs-Syndrom (MAS) ist eine gesonderte Entität des sekundären Hämophagozytose-Lymphohistiozytose-Syndroms (HLH), welches bei rheumatologischen Erkrankungen (6-10) wie auch beim KS auftreten kann und mit einer massiven systemischen Inflammation einhergeht. Die genaue Pathophysiologie des MAS ist unklar. Es wird vermutet, dass KS und MAS ähnlichen immunpathologischen Prozessen folgen, bei denen die Aktivierung von Makrophagen, T-Zellen und Histiozyten sowie proinflammatorischen Zytokinen und Chemokinen eine große Rolle spielen (11-13). Zu den klinischen Symptomen eines MAS gehören Fieber, Hepatosplenomegalie und eine Zytopenie (14). Auch kann es zu neurologischen Symptomen, Lymphadenopathie, Ödemen, Hautausschlägen oder einem Ikterus kommen (10). Laborchemisch zeigt sich vor allem ein stark erhöhtes Ferritin, sowie eine Erhöhungen von Triglyzeriden, Transaminasen und der Laktat-Dehydrogenase (LDH) wie auch eine Koagulopathie mit erniedrigtem Fibrinogen (15). Wichtig und wegweisend ist die Erhöhung des löslichen Interleukin-2-Rezeptors (sIL-2R), auch als lösliches CD25 bekannt (16). Die Hämophagozytose kann mitunter im Knochenmark oder Liquor nachgewiesen werden (17). Neben dem HLH-2004 Protokoll, das primär zur Diagnostik und Therapie des primären HLH (18) entwickelt wurde, werden in der Rheumatologie die Kriterien von Ravelli, die für die Diagnose des Übergangs einer systemischen juvenilen idiopathischen Arthritis (SJIA) in ein MAS ent- wickelt wurden, für die Diagnose eines MAS angewendet $(16,19)($ Tab. 1$)$.

Das MAS-KS wurde erstmals 1995 beschrieben (20). Es wird in der Literatur eine Inzidenz zwischen $1,1 \%$ (21) bis 1,9\% (13) angegeben, wobei Wang et al. (21) andeuten, dass das MAS-KS unterdiagnostiziert zu sein scheint. Es sind Fallberichte in der Altersspanne von sieben Wochen bis in die Adoleszenz beschrieben $(22,23)$. In ei-

\section{Klinische Kriterien des Kawasaki-Syndroms}

Fieber seit 5 Tagen ${ }^{1,2}$

+ mindestens 4 weitere Symptome:

Veränderungen an den Extremitäten

- Akut: Erytheme palmar und plantar, Ödeme an Händen und Füßen

- Subakut: Desquamation palmarl plantar in Woche 2 und 3

Polymorphes Exanthem

Bilaterale, nicht purulente Konjunktivitis

Veränderungen oropharyngeal

- Erythem, spröde Lippen, Erdbeerzunge, diffuse Injektion der oropharyngealen Schleimhäute

Zervikale Lymphadenopathie (Durchmesser $>1,5 \mathrm{~cm}$ ), meist einseitig + Ausschluss anderer Erkrankungen, die die Symptome kausal erklären können

1 Patienten mit Fieber von mindestens 5 Tagen und weniger als 4 von den oben aufgeführten klinischen Symptomen können als KS diagnostiziert werden, wenn in der 2-D-Echokardiographie oder Angiografie Koronararterienpathologien detektiert werden.

2 In Anwesenheit von $\geq 4$ Hauptkriterien kann das KS bereits am 4 . Krankheitstag diagnostiziert werden. Sehr erfahrene Kliniker, die bereits viele KS behandelt haben, können ggf. die Diagnose auch vor dem 4. Krankheitstag stellen. 
Tab. 1 MAS-Diagnosekriterien im Rahmen einer SJIA

\begin{tabular}{|c|c|c|}
\hline & Ravelli 2005 (19) & Ravelli 2016 (16) \\
\hline Klinik & $\begin{array}{l}\text { - ZNS-Dysfunktion } \\
\text { - Irritabilität } \\
\text { - Desorientierung } \\
\text { - Lethargie } \\
\text { - Kopfschmerzen } \\
\text { - Krampfanfälle } \\
\text { - Koma } \\
\text { - Blutungsneigung } \\
\text { - Purpura } \\
\text { - Hämatomneigung } \\
\text { - Schleimhautblutungen } \\
\text { - Hepatomegalie ( } \geq 3 \mathrm{~cm} \text { unter dem } \\
\text { Rippenbogen) }\end{array}$ & $\begin{array}{l}\text { Fieber } \\
\text { und } \\
\text { Verdacht auf oder bestätigte syste- } \\
\text { mische juvenile idiopathische Arthri- } \\
\text { tis }\end{array}$ \\
\hline Labor & $\begin{array}{l}\text { - Thrombozyten } \leq 262 \times 10 \mathrm{G}^{9} / \mathrm{l} \\
\text { - Aspartamino-Transferase }>59 \mathrm{U} / \mathrm{L} \\
\text { - Leukozyten } \leq 4,0 \times 10 \mathrm{G}^{9} / / \\
\text { - Fibrinogen } \leq 2,5 \mathrm{~g} / \mathrm{L}\end{array}$ & $\begin{array}{l}\text { - Ferritin }>684 \mu \mathrm{g} / \mathrm{l} \\
\text { - Thrombozyten } \leq 181 \times 10 \mathrm{G}^{9} / \mathrm{l} \\
\text { - Aspartamino-Transferase }>48 \mathrm{U} / \mathrm{L} \\
\text { - Triglyzeride }>156 \mathrm{mg} / \mathrm{dl} \\
\text { - Fibrinogen } \leq 3,6 \mathrm{~g} / \mathrm{L}\end{array}$ \\
\hline Histologie & $\begin{array}{l}\text { - Hämophagozytose im Knochen- } \\
\text { mark }\end{array}$ & \\
\hline Diagnosestellung & $\begin{array}{l}\text { MAS möglich, wenn: } \\
-\geq 2 \text { Laborkriterien } \\
\text { - } \geq 2 \text { klinische und/oder Laborkrite- } \\
\text { rien }\end{array}$ & $\begin{array}{l}\text { MAS möglich wenn: } \\
\text { 1.) Klinik + Ferritin }>684 \mu \mathrm{g} / \mathrm{l} \\
\text { 2.) }+2 \text { weitere Laborwerte }\end{array}$ \\
\hline Anmerkungen & $\begin{array}{l}\text { Knochenmarkspunktion ist nur in } \\
\text { unklaren Fällen nötig }\end{array}$ & \\
\hline
\end{tabular}

ner systematischen Übersichtsarbeit lag das Durchschnittsalter des MAS-KS bei 5,6 Jahren (24). Bei Jugendlichen scheint sich das MAS oft über ein atypisch verlaufendes KS zu manifestieren (25). Das MAS kann vor, mit und nach einem diagnostizierten KS auftreten (26). Vielfach überlappen sich jedoch die Symptome von MAS und KS (22) und führen unbehandelt zu einem lebensbedrohlichen Multiorganversagen (16). Die Therapie der Wahl eines MAS-KS sind intravenöse Hochdosis-Steroide und IVIG (7, 9, 24, 27, 28), aber auch Ciclosporin und Biologika (Interleukin-1-Rezeptor-Antagonisten, Tumornekrose-Faktor-Antagonisten) $(7,24,28)$. In refraktären Fällen kann diskutiert werden, ob analog zum HLHProtokoll Etoposid trotz der hohen Organtoxizität und der Knochenmarkssuppression eingesetzt wird $(7,24)$.

\section{Kasuistik}

Ein $64 / 12$ Jahre altes Mädchen wurde mit Halsschmerzen und hohem Fieber seit drei Tagen dem Kinderarzt vorgestellt. Auf Grund der Klinik und einem negativen

Tab. 2 Diagnostischer und laborchemischer Verlauf bei der Patientin

\begin{tabular}{|c|c|c|c|c|c|c|c|}
\hline & Tag 3 & Tag 4 & $\operatorname{Tag} 5$ & $\operatorname{Tag} 6$ & Tag 8 & Tag 15 & Tag 32 \\
\hline $\begin{array}{l}\text { Blutbild (Hb in } \mathrm{g} / \mathrm{l} \text {; } \\
\left.\mathrm{Tc} / \mathrm{Lc} \text { in } \times 10 \mathrm{G}^{9} / \mathrm{l}\right)\end{array}$ & & $\begin{array}{l}\text { Tc } 129 \\
\text { Lc } 4,8 \\
\text { Hb } 119\end{array}$ & $\begin{array}{l}\text { Tc } 88 \\
\text { Lc } 3,3 \\
\text { Hb } 102\end{array}$ & $\begin{array}{l}\text { Tc } 98 \\
\text { Lc } 4,3 \\
\text { Hb } 99\end{array}$ & $\begin{array}{l}\text { Tc } 129 \\
\text { Lc } 17,7 \\
\text { Hb } 92\end{array}$ & & $\begin{array}{l}\text { Tc } 295 \\
\text { Lc } 14 \\
\text { Hb } 132\end{array}$ \\
\hline CRP (mg/l) & & 189 & 267 & 271 & & 10,6 & 12 \\
\hline Ferritin $(\mu \mathrm{g} / \mathrm{l})$ & & & 770 & 923 & 937 & 336 & 37 \\
\hline AST (U/L) & & 37 & 42 & & 36 & 38 & 31 \\
\hline ALT (U/L) & & 25 & 28 & & 31 & 49 & 28 \\
\hline Triglyzeride (mg/dl) & & & & 280 & 352 & 177 & 155 \\
\hline Fibrinogen $(g / L)$ & & & & 4,94 & 7,47 & 3,91 & \\
\hline Echokardiografie & & & - Z-Score 1,9-3,5 & - Perikarditis & & normal & normal \\
\hline $\begin{array}{l}\text { Zusätzliche Diag- } \\
\text { nostik }\end{array}$ & - Strep. A - & $\begin{array}{l}\text { Sonografie: Aus- } \\
\text { schluss zervikale } \\
\text { Abszedierung }\end{array}$ & $\begin{array}{l}\text { - CMV - } \\
\text { EBV - } \\
\text { - Parvo B19 - } \\
\text { - respiratorisches } \\
\text { Panel - } \\
\text { - Mykoplasmen - }\end{array}$ & $\begin{array}{l}\text { - Lungenödem } \\
\text { - Pleuraergüsse bds. } \\
\text { - Aszites } \\
\text { - Gallenblasenhy- } \\
\text { drops } \\
\text { Knochenmark- } \\
\text { punktion: Hämo- } \\
\text { phagozytose }\end{array}$ & $\begin{array}{l}\text { - Liquor Zytologie } \\
\text { unauffällig } \\
\text { - sIL-2R } 2448 \text { pg/ } \\
\text { ml }\end{array}$ & & \\
\hline
\end{tabular}

Referenzwerte: CRP < 10 mg/l; Ferritin <55 g/l; AST 26-55 U/L; ALT 9-25 U/L; Triglyzeride 30-110 mg/dl; Fibrinogen 1,7-4,1 g/L, slL-2R <477 pg/ml Legende:Tc = Thrombozyten; Lc = Leukozyten; $\mathrm{Hb}=$ Hämoglobin; Respiratorisches Panel: Adenovirus, Influenza, Pertussis, Parapertussis, Mykoplasmen, Coronaviren, Metapneumovirus, Bocavirus, Parainfluenza, Legionellen, Rhino-Enterovirus, Rhinosynzytialvirus 
Streptokokken(Strep.)-A-Test wurde bei Verdacht auf eine virale Pharyngitis eine symptomatische Therapie begonnen. Am Folgetag präsentierte sich die Patientin bei Verschlechterung des Allgemeinzustands (AZ) in der Notaufnahme. Hier zeigten sich neben Fieber bis $40^{\circ} \mathrm{Celsius}$ und einer Pharyngitis, eine bilaterale Lymphadenopathie sowie eine Cheilitis. Laborchemisch fiel eine milde Bizytopenie bei stark erhöhtem CRP auf ( Tab. 2). Bei Verdacht auf eine bakterielle Lymphadenitis colli erfolgte eine zervikale Sonografie zum Ausschluss einer Abszedierung, und eine intravenöse antibiotische Therapie mit Amoxicillin/Clavulansäure $(150 \mathrm{mg} /$ $\mathrm{kgKG} / 24$ Stunden) wurde begonnen. Am fünften Fiebertag präsentierte sich die $\mathrm{Pa}$ tientin weiterhin in reduziertem AZ mit bilateraler, non-purulenter Limbus-aussparender Konjunktivitis und flüchtigem Exanthem. Im Labor wurde eine Panzytopenie bei steigenden Entzündungswerten detektiert ( Tab. 2). Die am Aufnahmetag abgenommenen Serologien zeigten negative Resultate ( Tab. 2). In der Echokardiografie zeigte sich eine Koronardilatation mit Z-Scores zwischen 1,9 und 3,5, sodass bei Verdacht auf ein komplettes KS eine leitliniengerechte Therapie mit Immunglobulinen (IVIG; 2 g/kgKG/12 Stunden) intravenös und Acetylsalicylsäure (ASS;
$83 \mathrm{mg} / \mathrm{kgKG} / 24$ Stunden) intravenös begonnen wurde ( $\mathrm{Abb}$. 1). Am sechsten Fiebertag kam es zu einer rapiden AZ-Verschlechterung mit respiratorischer Globalinsuffizienz und schwerer arterieller Hypotonie bei persistierendem Fieber, peripheren Ödemen und Erythem, sodass eine Verlegung auf die Intensivstation bei Multiorganversagen mit Intubation und Katecholamin-Unterstützung ( $\$$ Abb. 1$)$ notwendig wurde. In der weiteren Diagnostik ( $>$ Tab. 2) zeigte sich ein Lungenödem mit Pleuraergüssen, Aszites, ein Gallenblasenhydrops und eine vergrößerte Leber ohne Splenomegalie. In der Echokardiografie wurde eine reduzierte biventrikuläre Funktion bei Perikarditis und weiterhin moderat dilatierten Koronararterien diagnostiziert. Laborchemisch ( $\mathbf{T a b}$. 2) zeigten sich eine persistierende Panzytopenie, steigende Entzündungswerte, Hyperferritinämie und eine Hypertriglyzeridämie. Es erfolgte eine Knochenmarkpunktion, die eine Hämophagozytose zeigte und keine Hinweise auf eine Leishmaniose oder eine maligne Ursache ergab. Die Lumbalpunktion war unauffällig. Zusätzlich zeigte sich eine unauffällige Immunphänotypisierung der Lymphozyten. Die Analyse der NK-Zellen zeigte eine physiologische Perforin-Ausschüttung, sodass sich keine indirekten Hinweise für einen vorbestehenden Degranula- tionsdefekt ergaben. Bei Verdacht auf ein MAS-KS wurde noch am gleichen Tag eine Methylprednisolon-Pulstherapie mit $30 \mathrm{mg} /$ $\mathrm{kgKG} /$ Tag über drei Tage ( Abb. 1) begonnen. Zusätzlich erfolgte eine zweite Gabe von IVIG. Der am achten Tag bestimmte lösliche sIL-2R zeigte erhöhte Werte mit 2448 pg/ml (Referenz < 477 pg/ml). Im weiteren klinischen Verlauf zeigte sich eine kontinuierliche AZ-Verbesserung mit sich normalisierenden Entzündungswerten ( Tab. 2), sodass schrittweise die intensivmedizinische Therapie reduziert werden konnte und eine Extubation an Tag 12 möglich war ( Abb. 1). Die MethylprednisolonTherapie wurde von Tag 9 bis Tag 15 vorsichtig reduziert und dann gemäß HLHProtokoll auf Dexamethason in ausschleichender Dosierung umgestellt. In der Echokardiografie an Tag 15 zeigte sich bereits ein Normalbefund. Die ASS-Therapie wurde auf $5 \mathrm{mg} / \mathrm{kgKG} /$ Tag reduziert. Die Entlassung erfolgte an Tag 22, nachdem zuvor in einem Schädel-MRT Gefäßpathologien ausgeschlossen werden konnten. Von Tag 22 bis Tag 32 zeigte die Patientin palmar und plantar Desquamationen, passend zu einem KS ( Abb. 2). An Tag 38 konnte die Dexamethason-Therapie gestoppt werden und an Tag 42 wurde bei unauffälliger Echokardiografie die ASS-Therapie beendet.

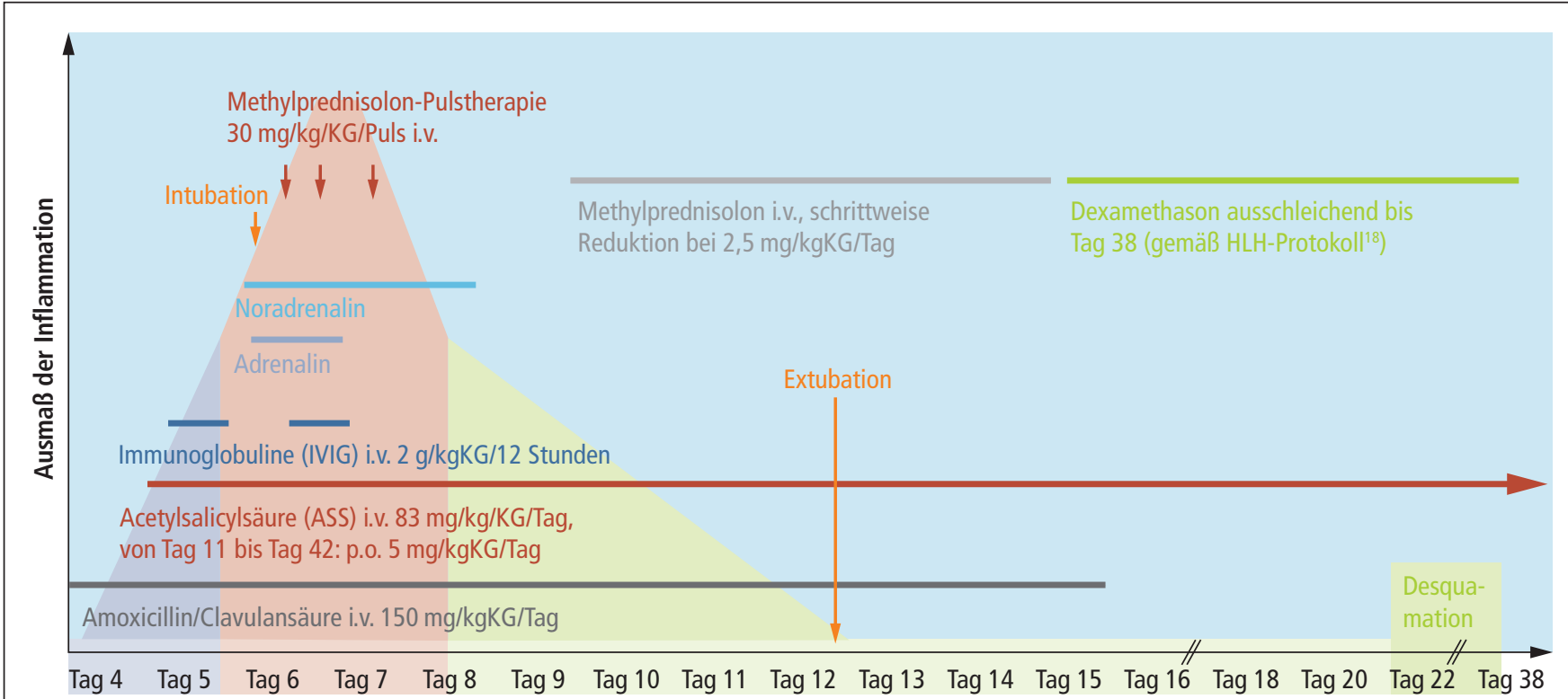

Abb. 1 Therapeutisches Management und Verlauf der Inflammation 


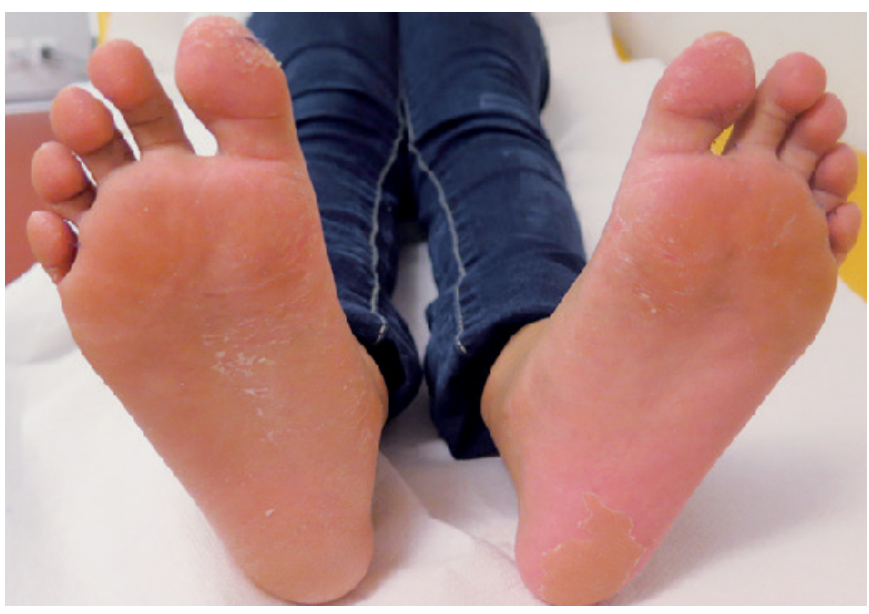

Abb. 2

Desquamation plantar

\section{Diskussion}

Unsere Patientin erfüllte am fünften Fiebertag alle Kriterien eines kompletten KS ( Kasten) (3). Mit Diagnosestellung des KS am fünften Krankheitstag wurde die empfohlene Therapie mit IVIG und ASS eingeleitet $(1,5)$. Am sechsten Krankheitstag verschlechterte sich unsere Patientin rapide trotz adäquater Therapie, wobei ein Nichtansprechen auf IVIG in $10-20 \%$ der Fälle beschrieben ist (3). Ein Risikofaktor für ein Nichtansprechen auf IVIG-Gabe scheinen bei Diagnosestellung vorhandene Koronardilatationen zu sein (29), die unsere Patientin bereits an Tag 5 aufwies. Zusätzlich scheinen stark erhöhte polymorphe Neutrophilen-Werte, deutlich erhöhtes natriuretisches Peptid B (NT-proBNP), ein hohes CRP und erhöhte Leberwerte (Aspartat-Aminotransferase [AST], ALT) mit einem Nichtansprechen assoziiert zu sein (30). Das Nichtansprechen auf IVIG wiederum scheint mit einem höheren Risiko für ein MAS vergesellschaftet zu sein (31). Aufgrund der rapiden AZ-Verschlechterung trotz rascher adäquater Therapie des KS bleibt zu vermuten, dass bei unserer $\mathrm{Pa}$ tientin bereits bei Diagnose des KS erste Frühzeichen für ein MAS bestanden (24, 26). Erschwerend kommt hinzu, dass einige Kardinalsymptome des KS gleichzeitig auch Symptome eines MAS sind (Fieber, Lymphadenopathie, Exanthem, reduzierter AZ) $(14,15)$, sodass ein Risiko für eine Verzögerung der Diagnose besteht.

\begin{tabular}{l|l|l}
\hline Parameter & Kasuistik & MAS-KS nach Garcia-Pavon (24) \\
\hline Hämoglobin & $\downarrow$ & \\
\hline Thrombozyten & $\downarrow$ & $\downarrow 48 / 69$ Patienten (87\%) \\
\hline Leukozyten & $\downarrow$ & \\
\hline CRP & $\uparrow$ & $\uparrow$ \\
\hline BSG & $\uparrow$ & $\uparrow 21 / 24$ Patienten (88\%) \\
\hline AST & $\rightarrow$ & $\uparrow 30 / 32$ Patienten (94\%) \\
\hline Fibrinogen & $\uparrow$ & $\downarrow 21 / 55$ Patienten (38\%) \\
\hline Ferritin & $\uparrow$ & $\uparrow 63 / 66$ Patienten (95\%) \\
\hline Triglyzeride & $\uparrow$ & $\uparrow 41 / 57$ Patienten (72\%) \\
\hline Koronardilatation & Ja & bei 32/69 Patienten (46\%) \\
\hline Splenomegalie & Nein & bei 45/65 Patienten (69\%) \\
\hline Fieber & Ja & bei 69/69 Patienten (100\%) \\
\hline
\end{tabular}

Legende: $\uparrow=$ erhöhter Parameter, $\downarrow=$ erniedrigter Parameter; $\rightarrow=$ im Referenzbereich
Tab. 3

Vergleich der in der Kasuistik beschriebenen Symptome mit in einer systematischen Übersichtsarbeit genannten Symptomen ( $n=69$ Patienten) im Rahmen eines MAS-KS

\section{Zusammenfassung}

Das Kawasaki-Syndrom (KS) ist eine akute febrile Vaskulitis der kleinen und mittelgroBen Arterien. Gefürchtet ist der Koronararterienbefall, der lebensbedrohlich sein kann. Für das KS scheint eine Immundysregulation in prädisponierten Personen ursächlich zu sein. Hochentzündliche Verläufe können sich als Makrophagenaktivierungs-Syndrom (MAS) manifestieren. Die Kasuistik schildert den Krankheitsverlauf eines sechsjährigen Mädchens mit KS, welches am fünften Fiebertag mit Immunglobulinen (IVIG) und Acetylsalicylsäure behandelt wurde. Am sechsten Fiebertag kam es zu einer raschen klinischen Verschlechterung mit Multiorganversagen. Aufgrund der klinischen Symptomatik, Panzytopenie, Hyperferritinämie, Hypertriglyzeridämie und Hämophagozytose im Knochenmark wurde ein MAS diagnostiziert. Es erfolgte eine Methylprednisolon-Stoßtherapie und eine zweite IVIGGabe mit deutlicher Besserung des klinischen Zustands und konsekutiver Normalisierung der systemischen Inflammation. Diese Kasuistik zeigt, dass eine klinische Verschlechterung bei KS trotz adäquater First-Line-Therapie an ein MAS denken lassen sollte, da sich durch gezielte Diagnostik und rasche Therapie das Mortalitätsrisiko senken lässt.

An Tag 6 zeigte unsere Patientin eine Panzytopenie, welche von Wang et al. (21) als Zeichen für ein MAS-KS angegeben wird. Auch zeigte die Patientin einen großen Teil der laborchemischen Merkmale, die für ein MAS-KS in einer systematischen Übersichtsarbeit von Garcia-Pavon et al. (24) beschrieben sind ( Tab. 3). Bei unserer Patientin wurden persistierend erhöhte BSG-Werte $(70 \mathrm{~mm} / \mathrm{h} ; 82 \mathrm{~mm} / \mathrm{h})$ dokumentiert, wohingegen bei einem MAS-SJIA häufig im Verlauf sinkende BSG-Werte - unter anderem aufgrund der deutlicheren Hypofibrinogenämie - gesehen werden (24).

Unsere Patientin zeigte ein gutes Ansprechen auf IVIG und MethyprednisolonStoßtherapie $(7,9,24,27)$. Damit zeigt sie analog zu den in der Literatur beschriebenen MAS-KS ein gutes Therapieanspre- 


\section{Schlussfolgerung}

Bei Patienten mit Krankheitssymptomen passend zu einem KS sollte bei rascher AZVerschlechterung, unabhängig, ob eine IVIG-Therapie bereits initiiert wurde, eine Evaluation auf das Vorliegen eines MAS erfolgen. Bei KS-Symptomen und Hyperferritinämie sollte eine weitere Diagnostik in Hinblick auf ein MAS-KS begonnen und eine Therapie mit Methylprednisolon und IVIG frühzeitig eingeleitet werden, um den Schweregrad der Morbidität und das Mortalitätsrisiko zu senken.

chen, ohne dass der Einsatz des mit deutlich höherer Organtoxizität und Knochenmarksuppression einhergehenden HLHProtokolls nötig war $(7,24)$ oder auf eine Therapie mit Interleukin-1-Rezeptor-Antagonisten oder Tumornekrose-Faktor-Antagonisten zurückgegriffen werden musste $(24,28)$.

\section{Interessenkonflikt}

Die Autorin gibt an, dass kein Interessenkonflikt besteht.

\section{Literatur}

1. McCrindle BW, Rowley AH, Newburger JW et al. Diagnosis, Treatment, and Long-Term Management of Kawasaki Disease: A Scientific Statement for Health Professionals From the American Heart Association. Circulation 2017; 135 (17): e927-e999.

2. Capittini C, Emmi G, Mannarino S et al. An immune-molecular hypothesis supporting infectious aetiopathogenesis of Kawasaki disease in children. European Journal of Immunology 2018; 48 (3): 543-545.

3. Newburger JW, Takahashi M, Gerber MA et al. Diagnosis, treatment, and long-term management of Kawasaki disease: a statement for health professionals from the Committee on Rheumatic Fever, Endocarditis and Kawasaki Disease, Council on Cardiovascular Disease in the Young, American
Heart Association. Circulation 2004; 110 (17): 2747-2771.

4. Tremoulet AH, Jain S, Chandrasekar D et al. Evolution of laboratory values in patients with Kawasaki disease. The Pediatric Infectious Disease Journal. 2011; 30 (12): 1022-1026.

5. Oates-Whitehead RM, Baumer JH, Haines L et al. Intravenous immunoglobulin for the treatment of Kawasaki disease in children. The Cochrane Database of Systematic Reviews 2003(4):CD004000.

6. Esteban YM, de Jong JLO, Tesher MS. An Overview of Hemophagocytic Lymphohistiocytosis. Pediatric Annals 2017; 46 (8): e309-e313.

7. Sen ES, Clarke SL, Ramanan AV. Macrophage Activation Syndrome. Indian Journal of Pediatrics 2016; 83 (3): 248-253.

8. Cron RQ, Davi S, Minoia F, Ravelli A. Clinical features and correct diagnosis of macrophage activation syndrome. Expert Review of Clinical Immunology 2015; 11 (9): 1043-1053.

9. Lin CI, Yu HH, Lee JH et al. Clinical analysis of macrophage activation syndrome in pediatric patients with autoimmune diseases. Clinical Rheumatology 2012; 31 (8): 1223-1230.

10. Janka GE. Hemophagocytic syndromes. Blood Reviews 2007; 21 (5): 245-253.

11. Dumont B, Jeannoel P, Trapes L et al. [Macrophage activation syndrome and Kawasaki disease: Four new cases]. Archives de Pediatrie 2017; 24 (7): 640-646.

12. Simonini G, Pagnini I, Innocenti L et al. Macrophage activation syndrome/Hemophagocytic Lymphohistiocytosis and Kawasaki disease. Pediatric Blood \& Cancer 2010; 55 (3): 592.

13. Latino GA, Manlhiot C, Yeung RS et al. Macrophage activation syndrome in the acute phase of Kawasaki disease. Journal of Pediatric Hematology/Oncology 2010; 32 (7): 527-531.

14. Atteritano M, David A, Bagnato G et al. Haemophagocytic syndrome in rheumatic patients. A systematic Review. European Review for medical and pharmacological Sciences 2012; 16 (10): 1414-1424.

15. Janka GE. Familial and acquired hemophagocytic lymphohistiocytosis. European Journal of Pediatrics 2007; 166 (2): 95-109.

16. Ravelli A, Minoia F, Davi S et al. 2016 Classification Criteria for Macrophage Activation Syndrome Complicating Systemic Juvenile Idiopathic Arthritis: A European League Against Rheumatism/American College of Rheumatology/Paediatric Rheumatology International Trials Organisation Collaborative Initiative. Arthritis \& Rheumatology 2016; 68 (3): 566-576.

17. Ravelli A. Macrophage activation syndrome. Current Opinion in Rheumatology 2002; 14 (5): 548-552.

18. Henter JI, Horne A, Arico M et al. HLH-2004: Diagnostic and therapeutic guidelines for hemo- phagocytic lymphohistiocytosis. Pediatric Blood \& Cancer 2007; 48 (2): 124-131.

19. Ravelli A, Magni-Manzoni S, Pistorio A et al. Preliminary diagnostic guidelines for macrophage activation syndrome complicating systemic juvenile idiopathic arthritis. The Journal of Pediatrics 2005; 146 (5): 598-604.

20. Ohga S, Ooshima A, Fukushige J, Ueda K. Histiocytic haemophagocytosis in a patient with Kawasaki disease: changes in the hypercytokinaemic state. European Journal of Pediatrics 1995; 154 (7): 539-541.

21. Wang W, Gong F, Zhu W et al. Macrophage activation syndrome in Kawasaki disease: more common than we thought? Seminars in Arthritis and Rheumatism 2015; 44 (4): 405-410.

22. Titze U, Janka G, Schneider EM et al. Hemophagocytic lymphohistiocytosis and Kawasaki disease: combined manifestation and differential diagnosis. Pediatric Blood \& Cancer 2009; 53 (3): 493-495.

23. Dogan V, Karaaslan E, Ozer S et al. Hemophagocytosis in the Acute Phase of Fatal Kawasaki Disease in a 4 Month-Old Girl. Balkan medical Journal 2016; 33 (4): 470-472.

24. Garcia-Pavon S, Yamazaki-Nakashimada MA, Baez $\mathrm{M}$ et al. Kawasaki Disease Complicated With Macrophage Activation Syndrome: A Systematic Review. Journal of Pediatric Hematology/Oncology 2017; 39 (6): 445-451.

25. Palazzi DL, McClain KL, Kaplan SL. Hemophagocytic syndrome after Kawasaki disease. The Pediatric Infectious Disease Journal 2003; 22 (7): 663-666.

26. Chen Y, Shang S, Zhang C et al. Hemophagocytic lymphohistiocytosis at initiation of kawasaki disease and their differential diagnosis. Pediatric Hematology and Oncology 2010; 27 (3): 244-249.

27. Thabet F, Bellara I, Tabarki B et al. [Ischemic colitis and hemophagocytosis complicating Kawasaki disease]. Archives de Pediatrie 2004; 11 (3): 226-228.

28. Carter SJ, Tattersall RS, Ramanan AV. Macrophage activation syndrome in adults: recent advances in pathophysiology, diagnosis and treatment. Rheumatology 2018; doi: 10.1093/rheumatology/key006. [Epub ahead of print]

29. Bar-Meir M, Kalisky I, Schwartz A et al. Prediction of Resistance to Intravenous Immunoglobulin in Children With Kawasaki Disease. Journal of the Pediatric Infectious Diseases Society 2018; 7 (1): 25-29.

30. Kim MK, Song MS, Kim GB. Factors Predicting Resistance to Intravenous Immunoglobulin Treatment and Coronary Artery Lesion in Patients with Kawasaki Disease: Analysis of the Korean Nationwide Multicenter Survey from 2012 to 2014. Korean Circulation Journal 2018; 48 (1): 71-79.

31. Muise A, Tallett SE, Silverman ED. Are children with Kawasaki disease and prolonged fever at risk for macrophage activation syndrome? Pediatrics 2003; 112 (6 Pt 1): e495. 